\title{
Facial Trustworthiness Perception Across the Adult Lifespan
}

Didem Pehlivanoglu ${ }^{1}$, Ph.D., Tian Lin ${ }^{1,}$ Ph.D., Nichole R. Lighthall ${ }^{2}$, Ph.D., Amber Heemskerk ${ }^{1}$, B.S., Alexandria Harber ${ }^{1}$, B.S., Robert C. Wilson ${ }^{3}$, Ph.D., Gary R. Turner ${ }^{4}$, Ph.D., R. Nathan Spreng ${ }^{5}$, Ph.D., \& Natalie C. Ebner ${ }^{1,6,7,8}$, Ph.D.

${ }^{1}$ Department of Psychology, University of Florida, Gainesville, FL, USA

${ }^{2}$ Department of Psychology, University of Central Florida, Orlando, FL, USA

${ }^{3}$ Department of Psychology, University of Arizona, Tucson, AZ, USA

${ }^{4}$ Department of Psychology, York University, Toronto, Canada

${ }^{5}$ Department of Neurology and Neurosurgery, McGill University, Montreal, Quebec, Canada

${ }^{6}$ Institute on Aging, University of Florida

${ }^{7}$ Florida Institute for Cybersecurity Research, University of Florida

${ }^{8}$ Evelyn F. and William L. McKnight Brain Institute, University of Florida

*****This manuscript is in press at the Journals of Gerontology, Series B: Psychological Sciences and Social Sciences******

Correspondence: Didem Pehlivanoglu, dpehlivanoglu@ufl.edu 5300 words in main text; 50 references; 2 Tables; and 1 Figure 


\begin{abstract}
Objectives: Trust is crucial for successful social interaction across the lifespan. Perceiver age, facial age and facial emotion have been shown to influence trustworthiness perception, but the complex interplay between these perceiver and facial characteristics has not been examined.
\end{abstract} Method: Adopting an adult lifespan developmental approach, 199 adults (aged 22-78 years) rated the trustworthiness of faces that systematically varied in age (young, middle-aged, older) and emotion (neutral, happy, sad, fearful, angry, disgusted) from the FACES Lifespan Database. Results: The study yielded three key results. First, on an aggregated level, facial trustworthiness perception did not differ by perceiver age. Second, all perceivers rated young faces as most trustworthy; and middle-aged and older (but not young) perceivers rated older faces as least trustworthy. Third, facial emotions signaling threat (fear, anger, disgust) relative to neutral, happy, and sad expressions, moderated age effects on facial trustworthiness perception.

Discussion: Findings from this study highlight the impact of perceiver and facial characteristics on facial trustworthiness perception in adulthood and aging and have potential to inform first impression formation, with effects on trait attributions and behavior. This publication also provides normative data on perceived facial trustworthiness for the FACES Lifespan Database. Keywords: Trust, FACES, Adult lifespan development, Emotion, Aging 


\section{Introduction}

Trust is crucial for successful relationships throughout the lifespan. Initial trust in others is influenced not only by behavior but by appearance (e.g., facial cues of trustworthiness). For instance, even brief exposures to faces were sufficient to result in trait inferences about trustworthiness (Willis \& Todorov, 2006), with behavioral consequences (e.g., in elections, prison sentences; Zebrowitz \& Montepare, 2015). A meta-analysis (Bailey \& Leon, 2019) suggests that mechanisms of age-related differences in facial trustworthiness perception are not likely to generalize to other types of trustworthiness judgments (e.g., financial trust). Thus, identifying characteristics that contribute to facial trustworthiness perception across the adult lifespan is particularly important as these factors can inform processes involved in first impression formation and its consequences on trait attributions and behavior.

Current knowledge about age-related differences in facial trustworthiness perception is limited. While some evidence suggests that older compared to young adults give (generally) higher facial trustworthiness ratings (Cassidy et al., 2021; Castle et al., 2012; Zebrowitz et al., 2017), other studies show age equivalency in facial trustworthiness perception (Cortes et al., 2019; Petrican et al., 2013). In addition to perceiver age effects, facial characteristics such as the age of or the emotion expressed in a face play a role in facial trustworthiness perception (Slessor et al., 2014; Todorov, 2008); with some inconsistency of the direction of these effects across studies; and close to nothing known about the interplay between these perceiver and facial characteristics.

Also, previous studies exclusively compared young and older adults regarding their facial trustworthiness perception (see Bailey \& Leon, 2019, for an overview), while effects in middleaged adults are understudied (Lachman, 2015). Inclusion of middle-aged individuals in the study of facial trustworthiness perception is warranted to capture the phenomenon across adulthood. 
The present study went beyond previous work, by asking young, middle-aged, and older adults to rate the trustworthiness of faces that systematically varied in age (young, middle-aged, older) and emotion (neutral, happy, sad, fearful, angry, disgusted). The faces were taken from the FACES Lifespan Database, a large standardized collection of naturalistic faces that cover the adult lifespan and systematically vary in age, gender, and emotion (see Ebner et al., 2010 for details). The primary aims were to determine $(i)$ the effect of perceiver age on facial trustworthiness perception; (ii) the interplay between perceiver age and facial age on facial trustworthiness perception, and (iii) the moderating role of facial emotion on this interplay. A secondary aim was the provision of picture-specific normative data on perceived facial trustworthiness for the FACES Lifespan Database.

\section{Effects of Perceiver Age}

Evidence suggests age-related differences in facial trustworthiness perception in adulthood. Relative to young adults, older adults rated untrustworthy faces as more trustworthy (Cassidy et al., 2021; Castle et al., 2012; Zebrowitz et al., 2013, 2017), and also were more likely to categorize untrustworthy faces as trustworthy (Cassidy et al., 2019). In fact, a meta-analysis by Bailey and Leon (2019) supports greater trustworthiness ratings for faces that convey untrustworthiness in older compared to young perceivers (but see Cortes et al., 2019; Lin et al., 2022, for evidence of age equivalency).

Greater facial trustworthiness perception among older adults may be driven by reduced sensitivity to negative information in aging (Cacioppo et al., 2011); an interpretation that is supported by neuroimaging evidence of lower insula (Castle et al., 2012) and amygdala (Lin et al., 2022; Zebrowitz et al., 2018) activity to untrustworthy faces in older than young adults. Further, older adults allocated more attention to trustworthy than untrustworthy faces (Petrican et 
al., 2013) and they had relatively greater reward network (i.e., caudate) activity to high than medium/low trustworthy faces, while their reward circuitry did not differentiate between medium and low trustworthy faces (Zebrowitz et al., 2018). Thus, we hypothesized that overall facial trustworthiness ratings would be greater among older than middle-aged or young adults (Hypothesis 1).

\section{Interplay Between Perceiver Age and Facial Age}

Shared group membership (such as belonging to the same age group) may influence facial trustworthiness perception (Cassidy et al., 2021); with this effect perhaps particularly relevant when evaluating unfamiliar others regarding their trustworthiness (Williams, 2001). For example, older compared to young adults showed a greater bias toward thinking that speakers from their own (i.e., older) vs. the other (i.e., young) age group were telling the truth in a lie detection task; and gave higher trustworthiness ratings for still photographs of own-age than other-age speakers (Slessor et al., 2014; but see Zebrowitz et al., 2013, 2018).

Thus, similar to the own-age bias in attention and memory (Strickland-Hughes et al., 2020), an own-age bias may be at work in facial trustworthiness perception. However, while the own-age bias in face recognition memory has been studied extensively (Rhodes \& Anastasi, 2012), its role in facial trustworthiness perception is not well understood. Given that trust-related decision making relies heavily on social expertise and memory for experiences (Frazier et al., 2019), which may be greater for (more familiar) own-age than (less familiar) other-age faces, we hypothesized higher facial trustworthiness ratings for own-age than other-age faces independent of perceiver age (Hypothesis 2).

\section{Effects of Facial Emotion}


Another factor that influences facial trustworthiness perception is the emotion expressed. Frank and Ekman (1997) argued that liars leak fear, disgust, and anger, and these leakages can be used to detect their lies. Research with young adults has shown that trustworthiness judgments are an extension of judgments made based on a continuum between positive and negative emotions (Todorov, 2008). For example, using computer animation, trustworthy faces that expressed happiness were perceived as happier than untrustworthy faces that expressed happiness; and accordingly, untrustworthy faces that expressed anger were perceived as angrier than trustworthy faces that expressed anger (Oosterhof \& Todorov, 2009).

Further, the literature suggests age-related challenges in emotion recognition, particularly for negative facial expressions (Hayes et al., 2020). For instance, older relative to young adults perceived less danger in faces at the high end of a dangerousness continuum, with no age-related differences for less dangerous-looking faces (Ruffman et al., 2006). This weaker responsiveness to negatively valenced faces in older adults may influence their perception of trustworthiness cues. In fact, age-related deficits in lie detection were driven by older adults' poorer recognition of negative facial expressions (Ruffman et al., 2012; Stanley \& Blanchard-Fields, 2008).

It is possible that both facial emotion detection and facial trustworthiness perception are improved for own-age relative to other-age faces, and particularly so in negatively valenced faces and for older adults. This may be because facial regions such as the eyes and mouth are diagnostic for both age (George \& Hole, 1998) and emotion (Sullivan et al., 2007) detection. Thus, drawing attention to those facial features by systematically varying them may affect facial trustworthiness ratings, and perhaps especially among older adults. Thus, we hypothesized that lower trustworthiness ratings for faces displaying negative compared to positive expressions was 
more pronounced for own-age than other-age faces in young, middle-aged, and particularly older perceivers (Hypothesis 3).

\section{Normative Data for the FACES Lifespan Database}

Some previous face databases report normative data on perceived trustworthiness. However, these databases either lack face images and raters that cover the adult lifespan ${ }^{1}$ or comprise faces across the adult lifespan but lack a fully-crossed design regarding facial age and emotion $^{2}$. To meet this need for normative data from an adult lifespan sample covering a wide age range of female and male faces that systematically vary in age and emotion, the present study added facial trustworthiness ratings for the FACES Lifespan Database (https://faces.mpdl.mpg.de/imeji/) for use in research.

\section{Method}

\section{Recruitment and Screening}

Two-hundred and six adults were recruited through Amazon Mechanical Turk (MTurk). Inclusion required MTurk workers to: $(i)$ have a proportion of previous MTurk tasks completed and approved > 98\%; (ii) have a number of previous MTurk tasks completed and approved > 1,000; (iii) understand written English; (iv) be able to see information on a computer screen; ( $v$ ) be 18-100 years old (to prevent self-misrepresentation, MTurk's built-in age qualification feature was used); (vi) use a laptop/desktop computer; (vii) be White/Caucasian who resides in the United States to reduce potential differences in perception of faces by racial category based on

\footnotetext{
${ }^{1}$ The Chicago Face Database (Ma et al., 2015) includes 158 faces with face models ranging from 18-40 years, rated by 1,087 participants with a mean age of 26.75 years; The Delaware Pain Database (Mende-Siedlecki et al., 2020) includes 229 faces with face models ranging from 18-34 years, rated by 616 participants with a mean age of 35.12 years; The Bogazici Face Database (Saribay et al., 2018) includes 264 faces with face models ranging from 19-32 years, rated by 1,207 participants with a mean age of 22.09 years.

${ }^{2}$ The Productive Aging Laboratory (PAL) Face Database (Minear \& Park, 2004) includes 576 faces with face models ranging from 18-93 years for which only a subset was rated on trustworthiness by participants from a somewhat restricted age range (e.g., 180 PAL faces were rated by 108 participants aged 18-54 years in Kennedy et al., 2009).
} 
evidence that race of face can influence face perception (Meissner \& Brigham, 2001) as well as facial trustworthiness evaluation (Cassidy et al., 2021) and given that all face models were White/Caucasian.

To enhance rigor of remote data collection, participants were randomly assigned to one of the counterbalancing conditions (see Face Rating Task below). Only after completion of the first study session were participants invited to complete the remaining two sessions to assure restriction of data collection to qualified participants. To prevent duplicate completion, participants provided their unique MTurk ID.

Further, to identify careless responding and ensure data quality, participants' data was only included if their session duration was within 2.5 standard deviations of the group average. This criterion led to removal of full data from 3 and partial data from 9 participants ( 7 from one session; 2 from two sessions). Also, following Curran (2016; also see Aguinis et al., 2021), data were removed if participants gave the same rating to $\geq 50 \%$ faces in a session. This criterion resulted in removal of full data from 4 and partial data from 3 participants (all from one session). Finally, chronological age was assessed twice (in a free format and as date of birth) to assure accuracy.

Table 1 presents characteristics of the final analysis sample, which included 199 adults of whom $79 \%$ had full data and $21 \%$ partial data from one (14\%) or two (7\%) sessions. Results from sensitivity analysis to determine the minimum detectable effect size with the available sample size are reported in the Supplemental Material.

Table 1 here

\section{Stimuli}


Stimuli were from the FACES Lifespan Database (Ebner et al., 2010), a large standardized database of naturalistic, high-quality colored still photographs of faces from 58 young (29 women; 19-31 years), 56 middle-aged (27 women; 39-55 years), and 57 older (29 women; 69-80 years) individuals (see Supplemental Material for samples). Each face identity in the database is represented in two parallel sets (Set A and Set B; 1,026 images in each set) of six prototypical emotions (neutral, happy, sad, fearful, anger, disgusted) for a total of 2,052 images. Images are $800 \times 1,000$ in pixel size, which corresponded to approximately $22 \times 27 \mathrm{~cm}$ when displayed on a 24-inch monitor.

\section{Procedure}

Study procedures were approved by the University of Florida Institutional Review Board. Data collection was conducted remotely via Qualtrics on MTurk. Participants electronically signed an informed consent; then filled out a short demographic profile and general health questionnaire (see Table 1). The study consisted of three sessions, completed on three separate days over the course of one week.

At the beginning of each session, participants responded to the Positive and Negative Affect Schedule (PANAS; Watson et al., 1988) indicating their present mood (To what extent do you feel [emotion adjective] right now?; 1 = Very slightly or not at all to 5 Extremely; e.g., Excited, Afraid; 10 positive and 10 negative adjectives). Next, participants rated a set of faces on facial trustworthiness (see Face Rating Task below). At the end of each session, participants indicated screen specifications of their device (e.g., size, resolution) and whether they encountered any technical difficulties. Additionally, at the end of the last session, participants filled out the 6-item General Trust Scale (Yamagishi \& Yamagishi, 1994) that assesses trust in other people (e.g., Most people are trustworthy; Most people are basically honest; 1 = Strongly 
disagree to $5=$ Strongly agree). Each session took about 1 hour. Participants received $\$ 4$ compensation at the end of each session and a $\$ 3$ bonus upon completion of all sessions.

\section{Face Rating Task}

Participants were instructed to sit $57 \mathrm{~cm}$ away from the computer screen for a comfortable viewing distance. They were informed that they would see various faces displaying different emotions and were asked to give their spontaneous personal judgment regarding the trustworthiness of each face (How trustworthy is this face?). In each trial, a color face image appeared in the center of the screen on a gray background with a sliding scale underneath from 0 $=$ Not at all trustworthy to $100=$ Extremely trustworthy. The task was self-paced with each face presented for at least 5 seconds. Once participants gave their response and at least 5 seconds had passed, they could proceed to the next face. A cue prompted participants to take a 5-minute break after rating half of the faces, and the program advanced only after this break.

Participants rated up to 1,026 faces selected either from the A or B set. Face presentation order was pseudorandomized with three lists in total (one per session, each containing 342 faces, 57 faces per emotion). Within each pseudorandomized list, each face identity was presented twice, each time displaying a different emotion. The same face identity and facial emotion were not repeated more than two times in a row. The order of faces within each pseudorandomized list for set A was reversed to create the lists for set B. Pseudorandomized lists were administered in a counterbalanced order across participants.

\section{Data Analysis}

We used multilevel model with cross-random effects, which considered crossclassification of perceivers and faces, and a nesting structure for repeated observations within perceivers and faces. This model allowed for $(i)$ ratings given by the same perceiver to be 
correlated across various face images; and (ii) dependencies among ratings for the same face image given by different perceivers.

The outcome variable was facial trustworthiness rating (continuous; from 0-100); Facial Age (categorical; 0 = young, 1 = middle-aged, 2 = older; within-subjects) and Facial Emotion (categorical; 0 = neutral, 1 = happy, 2 = sad, 3 = fearful, 4 = angry, 5 = disgusted; withinsubjects) served as face-level predictors; Perceiver Age (categorical; $0=$ young, $1=$ middleaged, 2 = older; between-subjects) served as a perceiver-level predictor. The model included all main effects and their interactions. We also considered the random intercepts of ratings for faces and perceivers to estimate the variability of mean ratings across faces and perceivers. Picture set, gender of the perceiver $($ male $=0$; female $=1$ ), mood, interpersonal trust, facial attractiveness rating (collected in an independent sample; Ebner et al., 2010), counterbalancing order, and total number of faces rated by each perceiver served as covariates.

The model can be denoted as:

$\mathrm{Y}_{\mathrm{ij}}=\beta_{0}+\beta_{1}$ FacialAge $_{\mathrm{i}}+\beta_{2}$ FacialEmotion $_{\mathrm{i}}+\beta_{3}$ FacialAge FacialEmotion $_{\mathrm{i}}+\beta_{4}$ PerceiverAge $_{\mathrm{j}}+$ $\beta_{5}$ FaceialAge $_{i} \times$ PerceiverAge $_{j}+\beta_{6}$ FacialEmotion $_{i} \times$ PerceiverAge $_{j}+\beta_{7}$ FacialAge $_{i} \times$

FacialEmotion $_{i} \times$ PerceiverAge $_{j}+\beta_{8}$ FaceSet $_{i}+\beta_{9}$ PerceiverGender $_{j}+\beta_{10}$ PerceiverPositiveAffect $_{j}$ $+\beta_{11}$ PerceiverNegativeAffect $_{j}+\beta_{12}$ PerceiverTrust $_{j}+\beta_{13}$ TrialNumber $_{j}+$ $\beta_{14}$ CounterbalanceOrder $_{\mathrm{j}}+\beta_{15}$ FacialAttractiveness $_{\mathrm{ij}}+\zeta_{\mathrm{i}}+\zeta_{\mathrm{j}}+\varepsilon_{\mathrm{ij}}$

We applied maximum likelihood for estimations of all model parameters. Wald tests determined significance of effects. To interpret significant interactions, we compared ( $\mathrm{z}$ tests for pairwise comparisons) and plotted predicted marginal means from the estimated model parameters, corrected for multiple comparisons (Bonferroni). All analyses were performed in Stata (v.16.1). 


\section{Results}

Table 2 summarizes means and standard deviations of facial trustworthiness ratings by Perceiver Age, Facial Age, and Facial Emotion. The Perceiver Age main effect was not significant $\left(\chi_{(2)}^{2}=0.64, p=0.727\right)$, indicating that overall facial trustworthiness ratings were similar between perceivers from different age groups. Thus, our data did not support Hypothesis 1 of differences by perceiver age in overall facial trustworthiness perception.

Table 2 here

The Facial Age main effect was significant $\left(\chi^{2}(2)=32.65, p<.001\right)$ and was qualified by a significant interaction with Perceiver Age $\left(\chi_{(4)}^{2}=238.17, p<.001\right)$. All perceivers rated young faces as most trustworthy. However, while young perceivers did not differ in their facial trustworthiness ratings for middle-aged and older faces $(z=0.27, p=.999)$, both middle-aged and older perceivers rated older faces as less trustworthy than middle-aged faces $(z \mathrm{~s}>2.56, p \mathrm{~s}<$ .049). These findings did not support Hypothesis 2 of an own-age bias in facial trustworthiness perception.

The Facial Emotion main effect was significant $\left(\chi_{(5)}^{2}=29899.12, p<.001\right)$ and was qualified by significant interactions with Perceiver Age $\left(\chi_{(10)}^{2}=1146.35, p<.001\right)$ and Facial Age $\left(\chi_{(10)}^{2}=174.66, p<.001\right)$. Further qualifying these effects was a significant interaction between Perceiver Age x Facial Age x Facial Emotion $\left(\chi_{(20)}^{2}=136.96, p<.001\right)$. To decompose this three-way interaction, we conducted separate follow-up analyses within each facial emotion. As depicted in Figure 1 across Panel A, B, and C, young, middle-aged, and older perceivers showed a comparable pattern in their facial trustworthiness ratings for neutral, happy, and sad faces: All perceivers rated young neutral, happy, and sad faces as more trustworthy than middleaged and older faces displaying these emotions ( $z \mathrm{~s}>3.34, p \mathrm{~s}<.005)$, with no differences in 
facial trustworthiness ratings between middle-aged and older faces for these emotions $(z \mathrm{~s}<2.23$, $p s>.155)$.

In contrast, there were differences in facial trustworthiness ratings for faces displaying fear, anger, and disgust, with these differences mostly between young vs. middle-aged/older perceivers. Comparable to the pattern described above for neutral, happy, and sad faces, young perceivers did not perceive differences in facial trustworthiness between middle-aged and older fearful and disgusted faces $(z \mathrm{~s}<2.49, p \mathrm{~s}>.077)$, but they perceived middle-aged and older fearful and disgusted faces as less trustworthy than young faces displaying these emotions (zs > 3.44, $p \mathrm{~s}<.003)$. Young perceivers' trustworthiness ratings for angry faces, however, did not vary by facial age $(z \mathrm{~s}<2.01, p s>.267)$.

Different from young perceivers, middle-aged and older perceivers' facial trustworthiness ratings for young, middle-aged, and older faces did not vary by fear, anger, and disgust display. Rather, both middle-aged and older perceivers rated older fearful, angry, and disgusted faces as least trustworthy and young faces displaying these emotions as most trustworthy $(z \mathrm{~s}>2.45, p \mathrm{~s}<$ .015), with middle-aged fearful, angry, and disgusted faces falling in between. That is, for older (but not for young or middle-aged) perceivers, lower facial trustworthiness ratings for faces displaying negative expressions were more pronounced for own-age (i.e., older) than other-age (i.e., young, middle-aged) faces. This pattern was in partial support of Hypothesis 3 that lower facial trustworthiness ratings for negative compared to positive facial expressions would be more pronounced for own-age than other-age faces in young, middle-aged, and particularly older perceivers.

Figure 1 here

\section{Discussion}


Extending previous work (Castle et al., 2012; Cortes et al., 2019; Petrican et al., 2013; Zebrowitz et al., 2013, 2017), we examined perceiver age, facial age, and facial emotion in their interplay on facial trustworthiness perception. The study yielded three key results. First, on an aggregated level, facial trustworthiness perception did not differ by perceiver age. Second, all perceivers rated young faces as most trustworthy; and middle-aged and older (but not young) perceivers rated older faces as least trustworthy. Third, facial emotions signaling threat (fear, anger, disgust) relative to neutral, happy, and sad expressions moderated age effects on facial trustworthiness perception. These findings advance understanding of facial trustworthiness perception across adulthood, as discussed next.

\section{Perceiver Age Equivalency in Facial Trustworthiness Perception}

Overall trustworthiness ratings were comparable across perceivers of different ages. This finding is consistent with Cortes et al. (2019) who found that young and older adults did not differ in their facial trustworthiness ratings of computer-generated faces that varied systematically in intensity of perceived trust (i.e., high, neutral, low). Similarly, using a subset of faces from the FACES Lifespan Database, Lin et al. (2022) found no differences between young and older adults in overall facial trustworthiness ratings. Our results extend this previous work to an adult lifespan sample by also including middle-aged perceivers.

Our results, however, contrast with findings of age-related differences in facial trustworthiness ratings (Cassidy et al., 2021; Castle et al., 2012; Zebrowitz et al., 2013, 2017). This divergence may be due to methodological differences across studies. In Cassidy et al. (2019), Castle et al. (2012), and Zebrowitz et al. (2017), young and older adults rated neutral faces that were pre-categorized as least vs. most trustworthy; and greater trustworthiness ratings by older compared to young perceivers were only observed for untrustworthy faces. The current 
study, in contrast, leveraged all faces available in the FACES Lifespan Database and thus used a set of faces that varied widely in age range and emotions. This larger variation of prominent facial features may have resulted in more similar facial trustworthiness ratings between perceiver age groups. Also, given the naturalistic set of faces used here, drawn from an average population (see Ebner et al., 2010, for details), ratings fell in the medium range of the trustworthiness dimension (see Table 2 for means ranging from 37 to 75 with an average of 61.5 ); and perhaps resulting in more comparable facial trustworthiness perception among perceivers of different ages.

\section{Evidence of a Young Facial Trustworthiness Effect}

All perceivers rated young faces as more trustworthy than middle-aged and older faces. This "young facial trustworthiness effect" could reflect trait attributions as a function of facial age. That is, young faces may be perceived as more naïve than middle-aged and older faces (Grühn et al., 2011), thus eliciting higher perceived trustworthiness. Additionally, while young perceivers did not differ in their facial trustworthiness ratings for middle-aged and older faces, both middle-aged and older perceivers rated older faces (i.e., own-age faces for older perceivers) as least trustworthy. This finding is interesting from a developmental perspective, as it could reflect a role of familiarity on facial trustworthiness perception (e.g., young perceivers may not be as experienced with middle-aged and older faces; Ebner et al., 2018).

Collectively, however, our findings do not support an own-age bias in facial trustworthiness perception, in line with findings from similar studies with still face images. Zebrowitz and colleagues (2013), for example, found no own-age bias in perceived trustworthiness but rather observed that older faces were perceived as more trustworthy than young faces (see also Zebrowitz et al., 2018). 
It is possible that more favorable facial trustworthiness perception of own-age than otherage faces is only present in dynamic task contexts rather than appearance-based evaluation. This suggestion is supported by evidence from a video-based lie/truth detection task with older adults in which a greater truth bias (i.e., being more likely to think that deceptive speakers were telling the truth) was observed for own-age than other-age speakers (Slessor et al., 2014). Similarly, studies using economic games with multiple interactions with unfamiliar trustees support an own-age bias in that older (relative to young) adults showed greater trust for older trustees (Bailey et al. 2015; Holm \& Nystedt, 2005). Such findings suggest that both facial appearance (e.g., facial age) and experience-based factors (e.g., social interactions) play a role in trustworthiness perception. The present study, however, involved facial trustworthiness ratings based solely on appearance. Future studies should test whether availability of different trustworthiness cues (e.g., still faces vs. experience-based factors such as actual payoffs) or frequency of interactions (e.g., passive vs. low vs. high) impact trustworthiness perception across contexts (e.g., financial decision making, lie detection) to determine domain-specificity or rather than generality of own-age biases in trustworthiness perception.

\section{Facial Emotions Signaling Threat Moderated the Interplay Between Perceiver Age and} Facial Age

Quite comparable across the different perceiver ages, young neutral, happy, and sad faces were perceived as more trustworthy than middle-aged and older faces displaying these emotions. In contrast, age-related differences in facial trustworthiness perception were observed for faces displaying fear, anger, and disgust. These findings suggest that age effects in facial trustworthiness perception may be at work for faces with a threat-signaling function. Additionally, perhaps the high emotional arousal associated with fear, anger, and disgust may 
have enhanced age-differential effects for these emotions. Future studies should follow up on this possibility by systematically varying emotional arousal levels in their effect on facial trustworthiness perception across the adult lifespan.

We expected that lower trustworthiness ratings for faces displaying negative expressions would be particularly pronounced for own-age compared to other-age faces, perhaps due to greater sensitivity to and more familiarity with own-age faces. There was no evidence for this prediction among young perceivers. For neutral, happy, sad, fearful, and disgusted faces, young perceivers rated middle-aged and older faces as equivalent in facial trustworthiness, while less trustworthy than young faces. Young perceivers' trustworthiness ratings of angry faces, however, did not vary by facial age. It is possible that high arousal and strong negative valence of angry faces overrides the impact facial age has on young perceivers' facial trustworthiness ratings. Processing of angry faces may be associated with a heightened startle response and larger pupil dilation as the social threat conveyed by angry faces triggers strong psychophysiological reactivity (Springer et al., 2007; Pehlivanoglu et al., 2014).

Quite different from young perceivers, both middle-aged and older perceivers gave comparable trustworthiness ratings for fearful, angry, and disgusted faces, with higher ratings for young, followed by middle-aged and then older faces expressing these emotions. Consistent with our prediction, these findings suggest that greater sensitivity to and more familiarity with ownage faces may have improved older adults' detection of threat-signaling facial emotions, which is shown to typically decline with age (Hayes et al., 2020). Perhaps older and middle-aged perceivers particularly distrust older faces when those faces express fear, anger, or disgust; a strategy that could serve the maintenance of a positive self-view, which contributes to successful aging (Baltes \& Baltes, 1990). For example, older adults were shown to distance themselves 
from their own-age group when their own-age group was depicted as negative (Lin et al., 2017). Our findings extend this phenomenon to facial trustworthiness perception. Future experimental studies could specifically probe the role of age-stereotypes on facial trustworthiness perception across adulthood.

Neuroimaging studies suggest that age-related differences in trustworthiness perception are associated with changes in recruitment of the reward neural circuitry (i.e., striatum, caudate). Cassidy et al. (2021), for example, found a stronger positive relationship between caudate activity and trustworthiness ratings among older than young adults for in-group (i.e., same-race) faces. Similarly, Fareri et al. (2022) found greater striatal activity in response to receiving investment from friends compared to strangers among young adults, with this pattern reversed in older adults. Following up on the current findings, future studies could probe differential involvement of the reward network in facial trustworthiness perception for negative compared to positive emotions for own-age vs. other-age faces across adulthood.

\section{Limitations}

Use of an adult lifespan sample in the current study constitutes an advancement over previous extreme age-group (i.e., young vs. older) comparisons (Castle et al., 2012; Zebrowitz et al., 2017). However, the current study employed a cross-sectional design. Future studies would benefit from adopting an adult lifespan longitudinal or cross-sequential design, in which facial trustworthiness ratings from the same different-aged individuals are collected over time and across age cohorts for capturing both inter- and intra-individual change in facial trustworthiness perception across adulthood.

The FACES Lifespan Database only included images from White/Caucasian face models and our sample only included White/Caucasian participants, limiting the representativeness of 
our sample and the generalizability of our findings. Sociodemographic factors including race are often overlooked in social-cognitive aging research (Hamilton et al., 2022), despite evidence that the race of a face can influence facial trustworthiness perception (Birkás et al., 2014). Identifying possible moderation effects of race of the perceiver and/or race of the face will constitute an important future direction and this information needs to be integrated into the FACES Lifespan Database.

\section{Theoretical and Practical Contributions and Broader Implications}

By integrating perceiver and facial characteristics as well as perceivers and faces of middle-aged individuals, our study goes beyond previous work in allowing the capture of crosssectional patterns on facial trustworthiness perception in adulthood. Research suggests that agerelated differences in trustworthiness perception change based on the domain of trust (financial vs. non-financial) and its measurement (self-report vs. behavior), as well as depending on the reliability of trustworthiness cues (facial appearance vs. experience through interactions; Bailey \& Leon, 2019). Thus, age-related differences in the perception of trustworthiness from facial stimuli may be domain-specific and do not generalize to other types of trustworthiness judgments. The present study advances knowledge on specific perceiver and face related characteristics that drive age-related differences in facial trustworthiness perception.

The majority of evidence suggests that age-related differences in perceived facial trustworthiness are driven by reduced attention and sensitivity to negative information (e.g., cues of untrustworthiness). Qualifying this literature, findings from our study suggest that processing own-age faces may improve older adults' sensitivity to negative threat-signaling facial emotions and reduce trustworthiness ratings to these faces. These results have potential to inform 
mechanistic research on the processes that underlie first impression formation as it relates to perceived facial trustworthiness across the adult lifespan.

Finally, going beyond previous face databases (Ma et al., 2015; Mende-Siedlecki et al., 2020; Minear \& Park, 2004; Saribay et al., 2018), this project is the first to provide picturespecific normative data on perceived trustworthiness of faces that systematically vary in age and emotion. Given that the FACES Lifespan Database is widely used around the world (e.g., in 2022 alone, 325 access requests were approved; for an estimated total of 1,800 approved users to date), the current study constitutes an important practical contribution to research on trustworthiness perception. 


\section{Funding}

This work was supported by the Department of Psychology, College of Liberal Arts and Science, University of Florida, the National Institute on Aging of the National Institutes of Health grants 1R01AG057764 and R01AG072658, and the Florida Department of Health Ed and Ethel Moore Alzheimer's Disease Research Program grant 22A12. The content is solely the responsibility of the authors and does not necessarily represent the official views of the National Institutes of Health.

\section{Acknowledgements}

The authors have no conflict of interest to disclose and have complied with APA ethical standards in human subjects research. The full set of data and analysis script can be found in the OSF repository (https://osf.io/evg5h/?view_only=0a66ef5855d24943813f159af49aa77f).

Data has not been previously distributed. The study was not preregistered. DP and NCE designed the study. DP and AHa collected the data. DP, TL, and NCE developed the formal analytical strategy. DP and AHe processed the data. DP and TL conducted analyses and reported the findings. DP, TL, and NCE wrote the Methods. DP, NCE, and NL wrote the introduction. DP, NCE, and NL wrote the discussion. All authors contributed to manuscript conceptualization and editing and approved the final manuscript. 


\section{References}

Aguinis, H., Villamor, I., \& Ramani, R. S. (2021). MTurk research: Review and recommendations. Journal of Management, 47(4), 823-837. https://doi.org/10.1177/0149206320969787

Bailey, P. E., \& Leon, T. (2019). A systematic review and meta-analysis of age-related differences in trust. Psychology and Aging, 34(5), 674-685. https://doi.org/10.1037/PAG0000368

Bailey, P. E., Slessor, G., Rieger, M., Rendell, P. G., Moustafa, A. A., \& Ruffman, T. (2015). Trust and trustworthiness in young and older adults. Psychology and Aging, 30(4), 977986. https://doi.org/10.1037/a0039736

Baltes, P. B., \& Baltes, M. M. (1990). Psychological perspectives on successful aging: The model of selective optimization with compensation. In Successful aging: Perspectives from the behavioral sciences. (pp. 1-34). https://doi.org/10.1017/CBO9780511665684.003

Birkás, B., Dzhelyova, M., Lábadi, B., Bereczkei, T., \& Perrett, D. I. (2014). Cross-cultural perception of trustworthiness: The effect of ethnicity features on evaluation of faces' observed trustworthiness across four samples. Personality and Individual Differences, 69, 56-61. https://doi.org/10.1016/j.paid.2014.05.012

Cacioppo, J. T., Berntson, G. G., Bechara, A., Tranel, D., \& Hawkley, L. C. (2011). Could an aging brain contribute to subjective well-being? The value added by a social neuroscience perspective. In A. Todorov, S. T. Fiske, \& D. A. Prentice (Eds.), Social neuroscience: Toward understanding the underpinnings of the social mind (pp. 249-262). Oxford 
University Press. https://doi.org/10.1093/acprof:oso/9780195316872.003.0017

Cassidy, B. S., Boucher, K. L., Lanie, S. T., \& Krendl, A. C. (2019). Age effects on trustworthiness activation and trust biases in face perception. The Journals of Gerontology: Series B, 74(1), 87-92. https://doi.org/10.1093/geronb/gby062

Cassidy, B. S., Hughes, C., \& Krendl, A. C. (2021). A stronger relationship between reward responsivity and trustworthiness evaluations emerges in healthy aging. Aging, Neuropsychology, and Cognition, 28(5), 669-686. https://doi.org/10.1080/13825585.2020.1809630

Castle, E., Eisenberger, N. I., Seeman, T. E., Moons, W. G., Boggero, I. A., Grinblatt, M. S., \& Taylor, S. E. (2012). Neural and behavioral bases of age differences in perceptions of trust. Proceedings of the National Academy of Sciences, 109(51), 20848-20852. https://doi.org/10.1073/pnas.1218518109

Cortes, D. S., Laukka, P., Ebner, N. C., \& Fischer, H. (2019). Age-related differences in evaluation of social attributes from computer-generated faces of varying intensity. Psychology and Aging, 34(5), 686. https://doi.org/10.1037/pag0000364

Curran, P. G. (2016). Methods for the detection of carelessly invalid responses in survey data. Journal of Experimental Social Psychology, 66, 4-19. https://doi.org/10.1016/j.jesp.2015.07.006

Ebner, N. C., Luedicke, J., Voelkle, M. C., Riediger, M., Lin, T., \& Lindenberger, U. (2018). An adult developmental approach to perceived facial attractiveness and distinctiveness. Frontiers in Psychology, 9, 561. https://doi.org/10.3389/fpsyg.2018.00561 
Ebner, N. C., Riediger, M., \& Lindenberger, U. (2010). FACES-A database of facial expressions in young, middle-aged, and older women and men: Development and validation. Behavior Research Methods, 42(1), 351-362. https://doi.org/10.3758/BRM.42.1.351

Fareri, D. S., Hackett, K., Tepfer, L. J., Kelly, V., Henninger, N., Reeck, C., ... \& Smith, D. V. (2022). Age-related differences in ventral striatal and default mode network function during reciprocated trust. NeuroImage, 256, 119267. https://doi.org/10.1016/j.neuroimage.2022.119267

Frank, M. G., \& Ekman, P. (1997). The ability to detect deceit generalizes across different types of high-stake lies. Journal of Personality and Social Psychology, 72, 1429-1439. https://doi.org/10.1037/0022-3514.72.6.1429

Frazier, I., Lighthall, N. R., Horta, M., Perez, E., \& Ebner, N. C. (2019). CISDA: Changes in integration for social decisions in aging. Wiley Interdisciplinary Reviews: Cognitive Science, 10(3), e1490. https://doi.org/10.1002/wcs.1490

George, P. A., \& Hole, G. J. (1998). The influence of feature-based information in the age processing of unfamiliar faces. Perception, 27(3), 295-312. https://doi.org/10.1068/p270295

Grühn, D., Gilet, A. L., Studer, J., \& Labouvie-Vief, G. (2011). Age-relevance of person characteristics: Persons' beliefs about developmental change across the lifespan. Developmental Psychology, 47(2), 376-387. https://doi.org/10.1037/a0021315

Hamilton, L. J., Gourley, A. N., \& Krendl, A. C. (2022). They Cannot, They Will Not, or We Are Asking the Wrong Questions: Re-examining Age-Related Decline in Social 
Cognition. Frontiers in Psychology, 13, 894522.

https://doi.org/10.3389/fpsyg.2022.894522

Hayes, G. S., McLennan, S. N., Henry, J. D., Phillips, L. H., Terrett, G., Rendell, P. G., ... \& Labuschagne, I. (2020). Task characteristics influence facial emotion recognition ageeffects: A meta-analytic review. Psychology and Aging, 35(2), 295. https://doi.org/10.1037/pag0000441

Holm, H., \& Nystedt, P. (2005). Intra-generational trust-a semi-experimental study of trust among different generations. Journal of Economic Behavior \& Organization, 58(3), 403419. https://doi.org/10.1016/j.jebo.2003.10.013

Kennedy, K. M., Hope, K., \& Raz, N. (2009). Life span adult faces: Norms for age, familiarity, memorability, mood, and picture quality. Experimental Aging Research, 35(2), 268-275. https://doi.org/10.1080/03610730902720638

Lachman, M. E. (2015). Mind the gap in the middle: A call to study midlife. Research in Human Development, 12(3-4), 327-334. https://doi.org/10.1080/15427609.2015.1068048

Lin, T., Ankudowich, E., \& Ebner, N. C. (2017). Greater perceived similarity between self and own-age others in older than young adults. Psychology and Aging, 32(4), 377-387. https://doi.org/10.1037/pag0000173

Lin, T., Pehlivanoglu, D., Ziaei, M., Liu, P., Woods, A. J., Feifel, D., ... \& Ebner, N. C. (2022). Age-Related Differences in Amygdala Activation Associated With Face Trustworthiness but No Evidence of Oxytocin Modulation. Frontiers in Psychology, 13, 838642. https://doi.org/10.3389/fpsyg.2022.838642 
Ma, D. S., Correll, J., \& Wittenbrink, B. (2015). The Chicago face database: A free stimulus set of faces and norming data. Behavior Research Methods, 47(4), 1122-1135. https://doi.org/10.3758/s13428-014-0532-5

Meissner, C. A., \& Brigham, J. C. (2001). Thirty years of investigating the own-race bias in memory for faces: A meta-analytic review. Psychology Public Policy and Law, 7, 3-35. https://psycnet.apa.org/doi/10.1037/1076-8971.7.1.3

Mende-Siedlecki, P., Qu-Lee, J., Lin, J., Drain, A., \& Goharzad, A. (2020). The Delaware pain database: A set of painful expressions and corresponding norming data. Pain Reports, 5(6). https://doi.org/10.1097/PR9.0000000000000853

Minear, M., \& Park, D. C. (2004). A lifespan database of adult facial stimuli. Behavior Research Methods, Instruments, \& Computers, 36(4), 630-633. https://doi.org/10.3758/BF03206543

Oosterhof, N. N., \& Todorov, A. (2009). Shared perceptual basis of emotional expressions and trustworthiness impressions from faces. Emotion, 9(1), 128. https://psycnet.apa.org/doi/10.1037/a0014520

Pehlivanoglu, D., Jain, S., Ariel, R., \& Verhaeghen, P. (2014). The ties to unbind: age-related differences in feature (un)binding in working memory for emotional faces. Frontiers in Psychology, 5, 253. https://doi.org/10.3389/fpsyg.2014.00253

Petrican, R., English, T., Gross, J. J., Grady, C. L., Hai, T., \& Moscovitch, M. (2013). Friend or foe? Age moderates time-course specific responsiveness to trustworthiness cues. The Journals of Gerontology. Series B, Psychological Sciences and Social Sciences, 68(2), 215-223. https://doi.org/10.1093/geronb/gbs064 
Rhodes, M. G., \& Anastasi, J. S. (2012). The own-age bias in face recognition: A meta-analytic and theoretical review. Psychological Bulletin, 138(1), 146-174. https://doi.org/10.1037/a0025750

Ruffman, T., Murray, J., Halberstadt, J., \& Vater, T. (2012). Age-related differences in deception. Psychology and Aging, 27(3), 543-549. https://doi.org/10.1037/a0023380

Ruffman, T., Sullivan, S., \& Edge, N. (2006). Differences in the way older and younger adults rate threat in faces but not situations. The Journals of Gerontology Series B: Psychological Sciences and Social Sciences, 61(4), 187-194. https://doi.org/10.1093/geronb/61.4.p187

Saribay, S. A., Biten, A. F., Meral, E. O., Aldan, P., Třebický, V., \& Kleisner, K. (2018). The Bogazici face database: Standardized photographs of Turkish faces with supporting materials. PloS One, 13(2), e0192018. https://doi.org/10.1371/journal.pone.0192018

Slessor, G., Phillips, L. H., Ruffman, T., Bailey, P. E., \& Insch, P. (2014). Exploring own-age biases in deception detection. Cognition \& Emotion, 28(3), 493-506. https://doi.org/10.1080/02699931.2013.839438

Springer, U. S., Rosas, A., McGetrick, J., \& Bowers, D. (2007). Differences in startle reactivity during the perception of angry and fearful faces. Emotion, 7(3), 516. https://doi.org/10.1037/1528-3542.7.3.516

Stanley, J. T., \& Blanchard-Fields, F. (2008). Challenges older adults face in detecting deceit: The role of emotion recognition. Psychology and Aging, 23(1), 24-32. https://doi.org/10.1037/0882-7974.23.1.24 
Strickland-Hughes, C. M., Dillon, K. E., West, R. L., \& Ebner, N. C. (2020). Own-age bias in face-name associations: Evidence from memory and visual attention in younger and older adults. Cognition, 200, 104253. https://doi.org/10.1016/j.cognition.2020.104253

Sullivan, S., Ruffman, T., \& Hutton, S. B. (2007). Age differences in emotion recognition skills and the visual scanning of emotion faces. The Journals of Gerontology Series B: Psychological Sciences and Social Sciences, 62(1), 53-60. https://doi.org/10.1093/geronb/62.1.p53

Todorov, A. (2008). Evaluating faces on trustworthiness: An extension of systems for recognition of emotions signaling approach/avoidance behaviors. Annals of the New York Academy of Sciences, 1124(1), 208-224. https://doi.org/10.1196/annals.1440.012

Watson, D., Clark, L. A., \& Tellegen, A. (1988). Development and validation of brief measures of positive and negative affect: The PANAS scales. Journal of Personality and Social Psychology, 54(6), 1063-1070. https://doi.org/10.1037//0022-3514.54.6.1063

Williams, M. (2001). In whom we trust: Group membership as an affective context for trust development. The Academy of Management Review, 26, 377-396. $\underline{\text { https://doi.org/10.2307/259183 }}$

Willis, J., \& Todorov, A. (2006). First impressions: Making up your mind after a 100-ms exposure to a face. Psychological Science, 17(7), 592-598. https://doi.org/10.1111/j.1467-9280.2006.01750.x

Yamagishi, T., \& Yamagishi, M. (1994). Trust and commitment in the United States and Japan. Motivation and Emotion, 18(2), 129-166. https://doi.org/10.1007/BF02249397 
Zebrowitz, L. A., Boshyan, J., Ward, N., Gutchess, A., \& Hadjikhani, N. (2017). The older adult positivity effect in evaluations of trustworthiness: Emotion regulation or cognitive capacity?. PloS One, 12(1), e0169823. https://doi.org/10.1371/journal.pone.0169823

Zebrowitz, L. A., Franklin, R. G., Hillman, S., \& Boc, H. (2013). Older and younger adults' first impressions from faces: Similar in agreement but different in positivity. Psychology and Aging, 28(1), 202-212. https://doi.org/10.1037/a0030927

Zebrowitz, L. A., \& Montepare, J. M. (2015). Faces and first impressions. In M. Mikulincer, P. R. Shaver, E. Borgida, \& J. A. Bargh (Eds.), APA handbook of personality and social psychology, Vol. 1. Attitudes and social cognition (pp. 251-276). American Psychological Association. https://doi.org/10.1037/14341-008

Zebrowitz, L. A., Ward, N., Boshyan, J., Gutchess, A., \& Hadjikhani, N. (2018). Older adults' neural activation in the reward circuit is sensitive to face trustworthiness. Cognitive, Affective and Behavioral Neuroscience, 18(1), 21-34. https://doi.org/10.3758/s13415$\underline{017-0549-1}$ 


\section{Table 1}

\section{Sample Characteristics and Inferential Statistics}

\begin{tabular}{|c|c|c|c|c|}
\hline & $\begin{array}{l}\text { Young } \\
(\mathrm{N}=95)\end{array}$ & $\begin{array}{l}\text { Middle-Aged } \\
\qquad(\mathrm{N}=58)\end{array}$ & $\begin{array}{l}\text { Older } \\
(\mathrm{N}=46)\end{array}$ & $\begin{array}{l}\text { Difference test } \\
\text { (Y vs M vs O) }\end{array}$ \\
\hline & $M / \%(S D)$ & $M / \%(S D)$ & $M / \%(S D)$ & $F / \chi^{2}$ Test \\
\hline Age (years) & $29.80(4.68)$ & $51.71(5.82)$ & $65.11(4.21)$ & $868.97 *$ \\
\hline Range & $22-39$ & $40-59$ & $60-78$ & \\
\hline Gender (female) & $27 \%$ & $65 \%$ & $70 \%$ & $31.9 *$ \\
\hline Education (years) & $16.00(2.54)$ & $15.31(2.52)$ & $15.15(2.33)$ & 2.37 \\
\hline Vision & $4.40(0.81)$ & $4.45(0.78)$ & $4.35(0.64)$ & 0.22 \\
\hline Cognition & $4.44(0.74)$ & $4.50(0.71)$ & $4.41(0.65)$ & 0.21 \\
\hline Health & $4.03(1.03)$ & $3.91(1.00)$ & $3.42(1.20)$ & $7.44 *$ \\
\hline
\end{tabular}

Note. $\mathrm{Y}=$ Young; $\mathrm{M}=$ Middle-Aged; $\mathrm{O}=$ Older; $\mathrm{SD}=$ Standard Deviation. Chi-square test was used for comparing differences between percentages; $F$-test was used for testing mean differences. Self-reported vision (How would you describe your overall vision?), cognitive functioning (How would you describe your overall cognitive functioning?), and health (How would you describe your overall health?) were measured on a scale ranging from 1 (Poor) to 5 (Excellent). $* p<.01$. 


\section{Table 2}

Means (Standard Deviations) of Facial Trustworthiness Ratings (from $0=$ Not at all trustworthy to 100 = Extremely trustworthy) by Facial Age and Facial Emotion for Young, Middle-Aged, and Older Perceivers

\section{Young Perceivers}

\section{Facial Emotion}

$\begin{array}{cccccccc} & & \text { Neutral } & \text { Happy } & \text { Sad } & \text { Fearful } & \text { Angry } & \text { Disgusted } \\ \text { Facial Age } & \text { Young } & 59(21) & 73(19) & 51(23) & 48(24) & 42(26) & 44(25) \\ & \text { Middle-Aged } & 55(22) & 70(21) & 49(23) & 46(24) & 42(25) & 41(25) \\ & \text { Older } & 53(23) & 70(21) & 49(24) & 45(26) & 43(25) & 40(26)\end{array}$

\section{Middle-Aged Perceivers}

\section{Facial Emotion}

\begin{tabular}{cccccccc}
\cline { 3 - 4 } & & Neutral & Happy & Sad & Fearful & Angry & Disgusted \\
Facial Age & Young & $55(22)$ & $75(20)$ & $54(21)$ & $52(23)$ & $42(22)$ & $44(22)$ \\
& Middle-Aged & $50(22)$ & $73(21)$ & $49(21)$ & $48(22)$ & $40(21)$ & $40(21)$ \\
& Older & $49(22)$ & $72(21)$ & $48(21)$ & $46(22)$ & $37(21)$ & $37(21)$
\end{tabular}

Older Perceivers

Facial Emotion

\begin{tabular}{cccccccc}
\cline { 3 - 5 } & & Neutral & Happy & Sad & Fearful & Angry & Disgusted \\
Facial Age & Young & $54(22)$ & $75(17)$ & $52(21)$ & $53(22)$ & $44(23)$ & $48(22)$ \\
& Middle-Aged & $51(22)$ & $72(18)$ & $50(20)$ & $50(21)$ & $42(22)$ & $45(21)$ \\
& Older & $50(23)$ & $72(19)$ & $49(21)$ & $47(21)$ & $41(22)$ & $41(21)$
\end{tabular}


Figure 1. Estimated marginal means for facial trustworthiness ratings by facial age and facial emotion in (A) young, $(B)$ middle-aged, and $(C)$ older perceivers. Solid black lines represent young faces, dotted black lines middle-aged faces, and dashed gray lines older faces. Error bars denote standard errors. The y-axis ranges from 35 to 80 to reflect the actual range of trustworthiness ratings selected by perceivers in this study; the theoretical range was from $0=$ Not at all trustworthy and $100=$ Extremely trustworthy.

(A) Young Perceivers

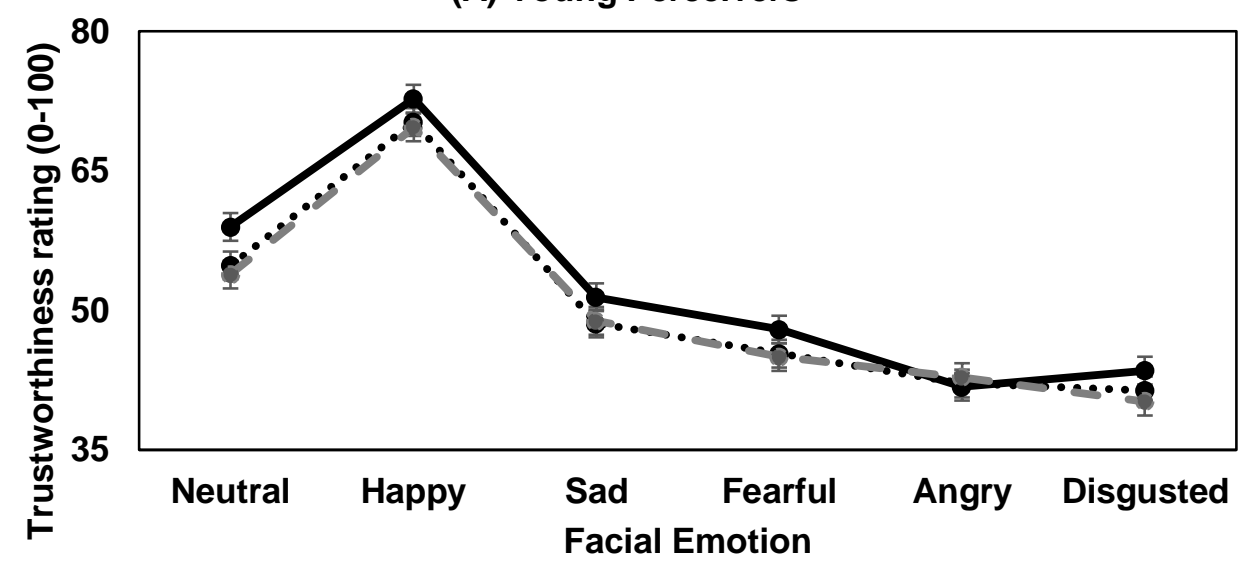

$\longrightarrow$ Young Faces $\quad \cdots \cdots .$. Middle-Aged Faces $\quad-\bullet$ Older Faces

(B) Middle-Aged Perceivers

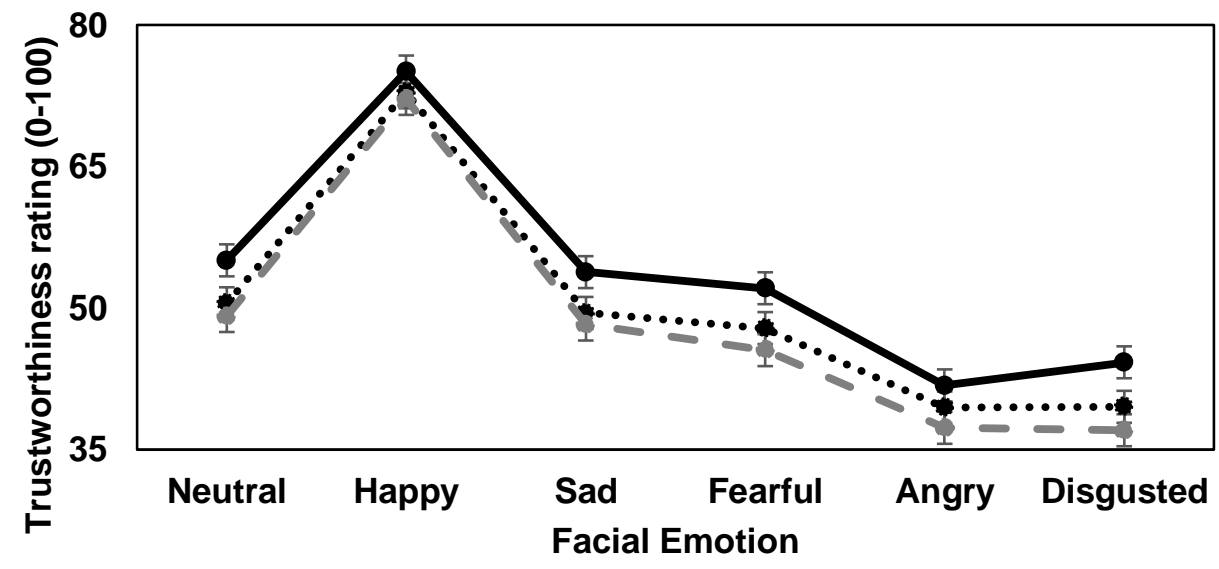

$\longrightarrow$ Young Faces $\cdots .$. Middle-Aged Faces $-\infty$ Older Faces

(C) Older Perceivers

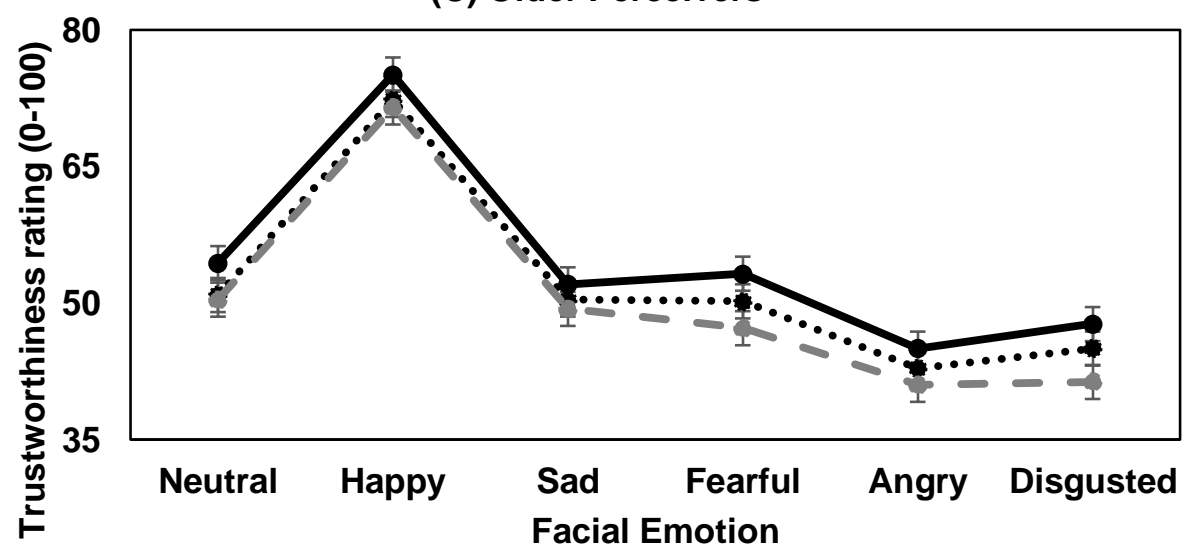

$\longrightarrow$ Young Faces $\cdots .$. Middle-Aged Faces $-\bullet$ Older Faces 